\title{
Permeability Estimation from Fracture Frequency by Indicator Simulation
}

\author{
Akira Kobayashi $^{1}$, Kiyohito Yamamoto ${ }^{2}$, Keisuke Inoue $^{3}$ and Shigeyasu Aoyama ${ }^{4}$
}

\begin{abstract}
The data on fracture frequency is easy to get at an in-situ investigation, while the permeability tests are not carried out so often because of financial limitation. Thus, it is very valuable to establish a technique to estimate the permeability distribution by using the data easily obtained like the fracture frequency. In this paper, an indicator simulation technique of the geostatistics was applied by using the fracture frequency as the soft data. To improve and develop the technique, the discrete fracture network generation method was applied to estimate the fracture frequency distribution. It was found as a result that it was effective to generate the permeability distribution by using only the fracture frequency when the number of permeability data was small and that of fracture frequency was large.
\end{abstract}

Keywords: Permeability; Fractured rock; Fracture frequency; Indicator simulation; Fracture network model

\section{Introduction}

It is a very important subject for geoenvironmental and groundwater pollution problems to know the permeability distribution of the ground. Estimation of the permeability distribution has a large effect on the prediction of solute transport because the advection and the mechanical dispersion are dependent on the velocity distribution. In particular, the dependency of mechanical dispersion on the distribution of permeability has been studied well (Gelhar, 1993; Dagan, 1989) . To understand the characteristics of mechanical dispersion, it is very important to know the correct distribution of permeability. If many in-situ permeability tests are carried out, the accuracy of the estimation becomes high. However, in-situ permeability tests are not carried out so often because of financial limitation. Thus, if the other kinds of data can be used to estimate the permeability distribution, it is very useful for geoenvironmental problems.

In general, the permeability of the ground is distributed with a given spatial correlation. Geostatistics is a useful method to make a heterogeneous permeability distribution according to such a correlation. Kriging, Cokriging, Gaussian Simulation and Indicator Simulation (Journel, 1989) have been used for hydrogeological problems. Kriging and Gaussian Simulation use only hard data, i.e., permeability data in this paper, while Cokriging and Indicator Simulation can use the other attributes, which are called soft data. In the case when the number of hard data is small, the method using the other attributes is very useful while the accuracy is very dependent on the soft data. Kobayashi et al. (2005) examined the elastic wave velocity, electric resistivity and geology as soft data to deduce the detail distribution of Lugeon value at the dam construction site. It was found as a

\footnotetext{
${ }^{1}$ Associate Professor, Graduate School of Agriculture, Kyoto University, Kitashirakawa-oiwake-cho, Sakyo-ku, Kyoto 606- 8502, Japan (Corresponding Author) E-mail; kobadesu@kais.kyoto-u.ac.jp

${ }^{2}$ Assistant, Graduate School of Agriculture, Kyoto University, Kitashirakawa-oiwake-cho, Sakyo-ku, Kyoto 606- 8502, Japan

${ }^{3}$ Researcher, National Institute for Rural Engineering, Tsukuba, Ibaraki 305-8609, Japan

${ }^{4}$ Professor, Department of Agriculture, Ishikawa Prefectural University, , Nonoichi, Ishikawa 921-8836, Japan
}

result that the accuracy of estimation was highest if both elastic wave velocity and geology were used as soft data.

The problem solved in this paper is related to the groundwater flow through a fractured rock mass.. For the agricultural facility, the dam construction, the pipe line construction and subsurface dam are related to the problem of groundwater flow through fractured rock mass. As the environmental problem related to the subsurface dam, transport of $\mathrm{NO}_{3}-\mathrm{N}$ through fractured rock mass is concerned to the quality of reserved groundwater (Isida et al., 2006). Moreover, water leaked from a degraded pipeline to the ambient environment through fractured media is also a potential problem. Therefore, estimation of permeability in the fractured media is important for agricultural activity.

The examination of geoenvironmental problem has to be effective and economical. In this paper, fracture frequency is used as soft data because the fracture frequency is easily measured. By using the fracture frequency data, the method to estimate the permeability distribution is examined. If this kind of method becomes suitable for practical use, examination of geoenvironmental problem becomes economical.

The method used in this study is called Indicator Simulation. This method uses Indicator Kriging and Cokriging to estimate the mean and variance of permeability at the location having no information on permeability. Then the permeability at the location is deduced by the assumption of the normal probability distribution. Multiple distributions of permeability are, therefore, produced by using the different seeds of random number generation. By using the multiple distributions of permeability having the same statistical property, advection-dispersion phenomena can be evaluated with solute tracking method by Monte Carlo simulation (Kobayashi et al., 1998). However, since the method to estimate the permeability distribution cannot be verified directly through the advection-dispersion problem, the multiple responses of interference hydraulic test using plural bore holes are simulated by Monte Carlo simulation and the accuracy of the simulation is discussed by the mean response and its $95 \%$ confidence range of averaged value in 


\section{this study.}

However, the correlation between fracture frequency and permeability is not so high in general. Thus the cumulative probability of permeability for a group of the fracture frequency might not be distinctive for that of the other group. In this case, the fracture frequency does not work effectively as soft data. In this paper, the relation between permeability and fracture frequency using the assumed statistical parameters is also examined. The statistical parameters are set such that the range of permeability has a clear difference among the groups of fracture frequency. Since this procedure is not the nonparametric statistics using the indicator of data, it can be considered as a parametric method using the assumed statistical parameter.

Moreover, the generation method of fracture geometric system has been developed in recent years (Dershowitz et al., 1991; Bonnet et al., 2001). At the test site in this study, the fracture geometry data are obtained, e.g., orientation, trace length and spacing. By making the statistical model of each geometric datum, the fracture network system can be statistically realized and the fracture frequency included in the element of the finite element analysis can be counted. The cumulative probability functions, used in Indicator Simulation, corresponding to the fracture frequency are applied for each element to estimate the permeability.

The interference test is simulated by using the generated permeability distributions and then the calculated hydraulic pressure responses at the observation points are compared with the measured ones. The effectiveness of the method is judged by the accuracy of the calculated responses.

\section{Indicator simulation method}

In this method, Indicator Kriging combining with Cokriging is carried out in order to get the cumulative probability distribution function of the permeability at the nonmeasured point. This is a method of liner regression with indicator $i$ of the measured hard data and indicator $y$ of the measured soft data. The detail of this method can be referred to Deutsch and Journel (1992).

$$
\begin{aligned}
I_{k}^{*}(\mathbf{u}) & =\left[\operatorname{Prob}\left\{z(\mathbf{u}) \leq z_{k}\left(n+n^{\prime}\right)\right\}\right]_{I K} \\
& =\lambda_{0} F\left(z_{k}\right)+\sum_{\alpha=1}^{n} \lambda_{\alpha}\left(\mathbf{u} ; z_{k}\right) i\left(\mathbf{u}_{\alpha} ; z_{k}\right) \\
& +\sum_{\alpha^{\prime}=1}^{n^{\prime}} v_{\alpha^{\prime}}\left(\mathbf{u} ; z_{k}\right) y\left(\mathbf{u}_{\alpha^{\prime}} ; z_{k}\right)
\end{aligned}
$$

where $I_{k}^{*}(\mathbf{u})$ is the probability that $z$ is smaller than $z_{k}$ at location $\mathbf{u}$. $\mathbf{u}$ is the position vector. In this study, $z$ means the permeability. $F$ is the probability that measured $z$ is smaller than $z_{k}$, which is obtained directly from the measured data. In this study, since the number of measured data is not enough, $F$ is assumed to be homogeneous for entire region. $\lambda_{0}$ is the weight of $F, n$ is the number of the hard data, and $n^{\prime}$ is the number of the soft data. $\mathbf{u}_{\alpha}$ is the position vector where the hard data are measured and $\mathbf{u}_{\alpha^{\prime}}$ is the one where the soft data are measured. The hard indicator data $i$ originated from the hard data $z\left(\mathbf{u}_{\alpha}\right)$ is given by

$$
\begin{array}{ll}
i\left(\mathbf{u}_{\alpha} ; z_{k}\right)=1 & \text { if } z\left(\mathbf{u}_{\alpha}\right) \leq z_{k} \\
i\left(\mathbf{u}_{\alpha} ; z_{k}\right)=0 & \text { if not }
\end{array}
$$

This means that if the measured permeability is smaller than a value of permeability $z_{k}$ indicator $i$ equals 1 , if not, $i$ becomes $0 . z_{k}(k=1, \ldots, K)$ are called the cutoff value. $K$ is the number of cutoff values. The soft indicator $y\left(\mathbf{u}_{\alpha^{\prime}}, z_{k}\right)$ is given by

$$
y\left(\mathbf{u}_{\alpha^{\prime}} ; z_{k}\right)=\operatorname{Prob}\left\{z\left(\mathbf{u}_{\alpha^{\prime}}\right) \leq z_{k}\right\}
$$

which is the probability that $z$ inferred from the soft data is smaller than cutoff value $z_{k}$. The probability is obtained from the scattergram, which is the plot between the hard and soft data measured at the same point. The soft data are divided into a few groups, and the probability $y$ is calculated for each $z_{k}$ in each group. In this paper, the scattergram is obtained from the relation of the permeability to the fracture frequency as shown in Figure1.

$\lambda_{\alpha}$ are the weights applied to the $n$ permeability samples, and $v_{\alpha^{\prime}}$ are the weights applied to the $n^{\prime}$ soft data. These are obtained by minimizing the square error of estimated values and are a function of the covariance matrix of hard and soft indicators. The covariance of the hard indicator is used for that of soft indicator for simplicity in many cases. The same treatment is carried out in this study. As mentioned later, this is because the number of data on the soft indicator was not obtained enough to estimate the covariance at the test site. Therefore, the spatial correlation considered in this study is dependent on the locations of measurement of permeability. The variogram of hard indicator is estimated by fitting to the exponential model.

In Indicator Simulation, the probability at non-measurement point is estimated by Cokriging of the equation (1). The probability that $z$ is smaller than the cutoff value $z_{k}$ is put in order of $z_{k}$, so that the cumulative probability at the location is given as shown in Figure 2. A random number is, then, generated from 0 to 1 , and the permeability corresponding to the random number is obtained from the cumulative probability distribution. Then, the same processes are carried out at the next non-measurement point to estimate the permeability. Thus, we can make multiple heterogeneous media by means of the different seed of the random number and the different turn of the non-measurement points. In this paper, 30 realizations are generated and the results are examined with the averaged values.

\section{In-situ test}

\subsection{Test site}

The method examined in this paper is applied to in-situ hydraulic tests. The in-situ experiment was carried out at Kamaishi mine (Yokoi et al., 1991). Figure 3 shows the location of test site. Figure 4 indicates schematic view of location of boreholes. The geology is Kurihashi granite and the depth is about $260 \mathrm{~m}$. Four boreholes, KH-4, 5, 6, 7 were dug in the horizontal direction from the tunnel KD-89. The length of each borehole was about $60 \mathrm{~m}$. KH-4, 5, 6 were set alongside in the tunnel axis direction and $\mathrm{KH}-7$ 


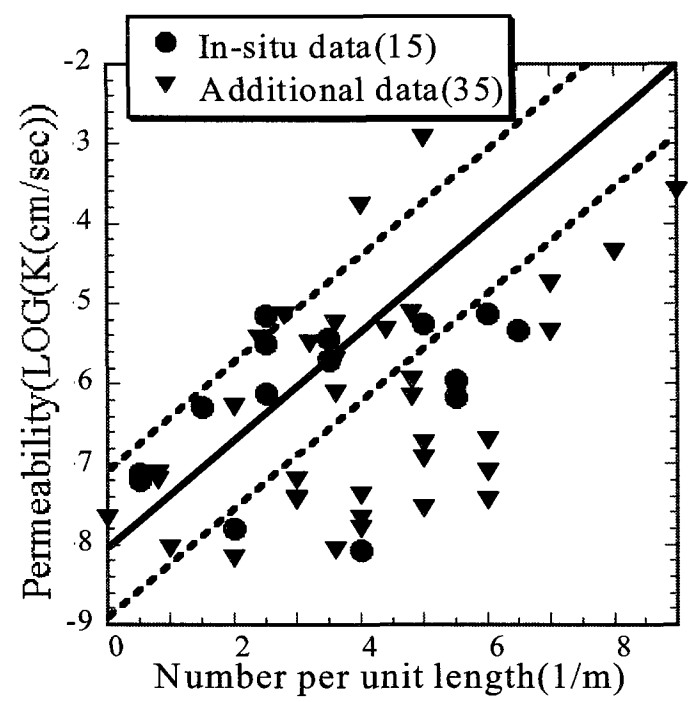

Figure1: Scattergram of permeability and fracture frequency

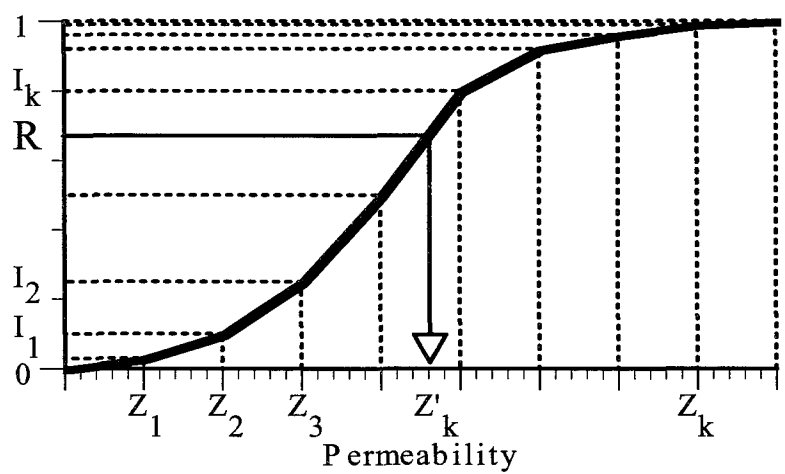

$\mathrm{R}$ :random number

Z'k:the estimated value

Figure2: Cumulative probability distribution and estimation of the permeability

was dug just above KH-5. The distance of each borehole is about $12 \mathrm{~m}$. The normal direction of main fractures was mostly parallel to the borehole direction.

The fracture observation was carried out at each borehole by borehole television camera, and the fracture density was obtained at each borehole. Furthermore, the single-hole hydraulic tests are carried out at 15 points of $\mathrm{KH}-5$, and the interference tests are also carried out by injecting from $\mathrm{A}$ of $\mathrm{KH}-5$ and observing at 12 points of $\mathrm{KH}-4, \mathrm{KH}-6$, and $\mathrm{KH}-7$ shown in Figure 4 (Okuno, 1994). Since the spatial correlation of permeability is obtained from the data of $\mathrm{KH}-15$, the anisotropic characteristics of spatial distribution cannot be evaluated in this study. Moreover, the permeability of each element is estimated as an isotropic parameter. This is because there is no information on the anisotropy of the permeability.
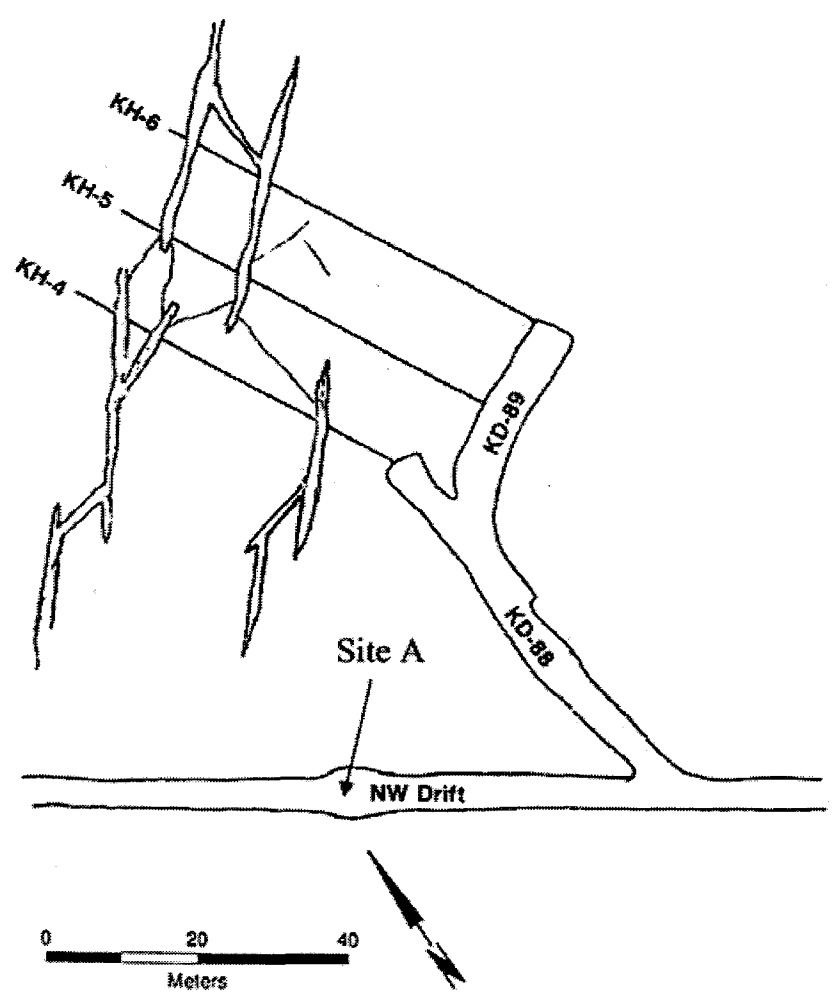

Figure 3: Location of test site and boreholes

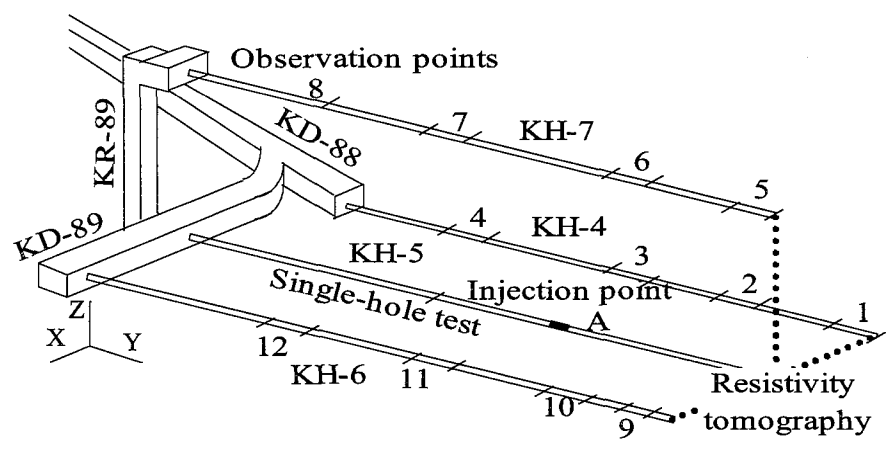

Figure 4: Location of each borehole and injection and observation points of interference test

$\mathrm{KH}-4$

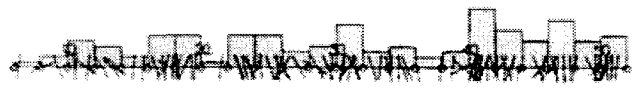

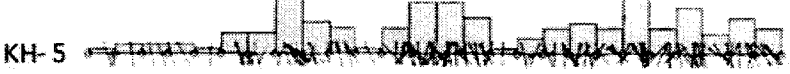

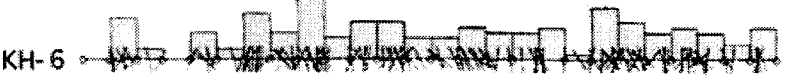

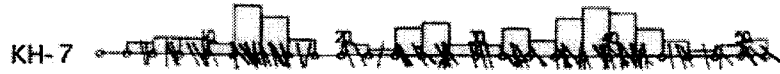

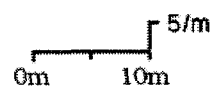

Figure 5: Observed fracture frequency at the boreholes 


\subsection{Scattergram}

Figure 5 is the result of the fracture frequency observation. The observation interval was about $2 \mathrm{~m}$. The histogram of each interval was shown in the figure. While the number of measurement point was is 100 , the number of intervals where both permeability and fracture frequency are investigated was only 15 . To overcome the shortage of the information, the data obtained at the other site in the same mine are also included. Test results at Site A shown in Figure 3 (Yokoi et al., 1991) are added to make the scattergram. At Site A, 35 tests were carried out. Figure 1 is the obtained scattergram in which totally 50 data are plotted. For the scattergram, the cutoff values of permeability are set by every order from $10^{-8} \sim 10^{-3} \mathrm{~cm} / \mathrm{sec}$. The fracture frequency is classified by the threshold values of $2,4,5.5$ and 6.5 number of fractures $/ \mathrm{m}$. $y$ of the equation (3) is calculated in each group of the soft data. Using the above information, Indicator Simulation is carried out. As mentioned above, the covariance of the hard indicator is used for the soft indicator. This is because the covariance of the soft indicator cannot be evaluated as a function of distance since the soft indicator is estimated from the scattergram including the data obtained from the other site.

\subsection{Simulation of interference test}

The analysis region is $44 \mathrm{~m} \times 60 \mathrm{~m} \times 32 \mathrm{~m}$ rectangular solid containing the margin of about $10 \mathrm{~m}$ from the edge of the boreholes. The region is divided into cube elements whose side is $2 \mathrm{~m}$. The total pressure at all the boundaries is fixed at $400 \mathrm{KPa}$. The boundary conditions are decided from the in-situ survey results. The injection rate at the point $\mathrm{A}$ of $\mathrm{KH}-5$ is fixed at $4.446 \times 10^{-6} \mathrm{~m}^{3} / \mathrm{sec}$, and the responses at observation points are simulated. While the other methods to evaluate the distribution of permeability than Indicator Simulation method, which are explained in the following section, are also examined, the isotropic permeability is distributed heterogeneously for entire region. The specific storativity is set at $3 \times 10^{-6} \mathrm{~m}^{-1}$ for all elements, which is the product of average porosity and water compressibility. The comparison between the methods is carried out by the error between calculated and measured transient responses at 10 observation points except for points 4 and 12 in Figure 4, at which the clear responses were monitored.

\section{Examination}

\subsection{Cases}

Based on the Indicator Simulation technique mentioned above, the permeability distribution is examined. However, as shown in Figure 1, the variance at each group of fracture frequency is relatively large. This means that the realization of permeability in a group is in a large range of permeability and the difference of the realized permeability between the groups is small.

To emphasize the effect of soft data, the relation between permeability and fracture frequency is assumed as shown in Figure1 by solid and dotted lines, in which solid line indicates the mean relation and dotted line shows the range of standard deviation. The gradient is inferred from the entire relation and the permeability of rock sample having no fracture, and the mean relation is obtained by shifting so that the mean measured fracture frequency in $\mathrm{KH}-5$ coincides with the mean measured permeability in the same borehole. The standard deviation is obtained from the measured permeability data in KH-5. By using this procedure, the relation between the permeability and fracture frequency gives the large permeability with increase of fracture frequency as shown in Figure 1. While it is not assured if this procedure is appropriate, the difference between non parametric method and parametric method using the assumed parameters can be examined.

Moreover, the fracture geometry information is obtained at Site A shown in Figure 3. Table 1 shows the inferred fracture stochastic data. By using the information, the fracture can be generated with discontinuous fracture network approach for the test site (Okuno, 1994). It is assumed that the length of fracture has a normal distribution and the dip direction has a Fisher distribution. The shape of fracture is assumed to be circular. The center of fracture is distributed randomly and the number of fracture in entire region is constrained by the area/volume in Table 1 . The measured fracture frequency is assumed to be a fracture density (the number of fracture per volume) of the corresponding element and is conditioned to the element.

By considering the number of fracture per volume as the fracture frequency, the fracture frequency of each element can be estimated from the generated fracture distribution. The permeability at each element is then generated with the estimated fracture frequency and the relation between the permeability and fracture frequency using the assumed statistic parameters, while the spatial correlation of hard and soft data is neglected. In this case, the measured permeability is difficult to be conditioned. Thus, Indicator

Table 1: Stochastic data of fracture geometry

\begin{tabular}{lccccc}
\hline Cluster Number & 1 & 2 & 3 & 4 & 5 \\
\hline Area/Volume $\left(\mathrm{m}^{-1}\right)$ & 0.85 & 0.74 & 0.40 & 0.63 & 0.52 \\
Mean length $(\mathrm{m})$ & 1.67 & 1.64 & 1.92 & 2.13 & 1.86 \\
St.dev. of length $(\mathrm{m})$ & 1.36 & 0.62 & 1.30 & 0.68 & 1.13 \\
Mean Dip direction $\left(^{\circ}\right)$ & 317 & 249 & 179 & 180 & 353 \\
Mean Dip ( $\left.{ }^{\circ}\right)$ & 70 & 89 & 77 & 16 & 76 \\
Fisher k $\left(^{\circ}\right)$ & 14 & 7 & 31 & 8 & 13 \\
\hline
\end{tabular}

Table 2: Examination cases

\begin{tabular}{ll}
\hline Case & \multicolumn{1}{c}{ Method } \\
\hline 1 & $\begin{array}{l}\text { Indicator Simulation using scattergram and conditioned } \\
\text { permeability data }\end{array}$ \\
\hline 2 & $\begin{array}{l}\text { Indicator Simulation using assumed relation between } \\
\text { permeability and fracture frequency and conditioned } \\
\text { permeability data }\end{array}$ \\
\hline 3 & $\begin{array}{l}\text { Indicator Simulation using assumed relation between } \\
\text { permeability and fracture frequency without condi- } \\
\text { tioned permeability data }\end{array}$ \\
\hline 4 & $\begin{array}{l}\text { Permeability generated by using assumed relation be- } \\
\text { tween permeability and fracture frequency and gener- } \\
\text { ated fracture frequency with discontinuous fracture } \\
\text { network technique }\end{array}$ \\
\hline
\end{tabular}


Simulation without conditioned permeability data is also examined in order to compare with the case using the discontinuous fracture network approach. As a consequence, four cases, as shown in Table 2, are examined in this study.

\subsection{Examination results}

Figure 6 shows the examples of the permeability distributions for each case. The value is a logarithm of permeability of $\mathrm{cm} / \mathrm{sec}$. The plane is a horizontal one through the $\mathrm{KBH}-4$, 5 and 6 . The cases using the Indicator Simulation approach, Cases 1, 2 and 3, show similar distribution, while Case 4 indicates quite different distribution. This is because Case 4 does not take into account any spatial correlation of permeability and fracture frequency. The similarity among Cases 1 to 3 is caused from the spatial correlation of fracture frequency.

By generating about 30 patterns for each case, the $95 \%$ confidence range of averaged value of the responses at the observation points at each time step are compared with the measured responses. Table 3 shows the number of hard and soft data used in each case and the total error of simulation results of each case. The total error is calculated by the square error between the measured and calculated transient responses at 10 observation points. Figure 7 indicates the error of each case for each observation point except for points 4 and 12 points at which the measured data were lacked due to unclear responses. The large variation among cases can be seen at points $2,3,6,7$ and 11. At those observation points, relatively large errors are observed. It is, however, noticeable that the error is very small such as a few centimeters. The accuracy discussed in this paper is very high in comparison with the ordinary seepage problems.

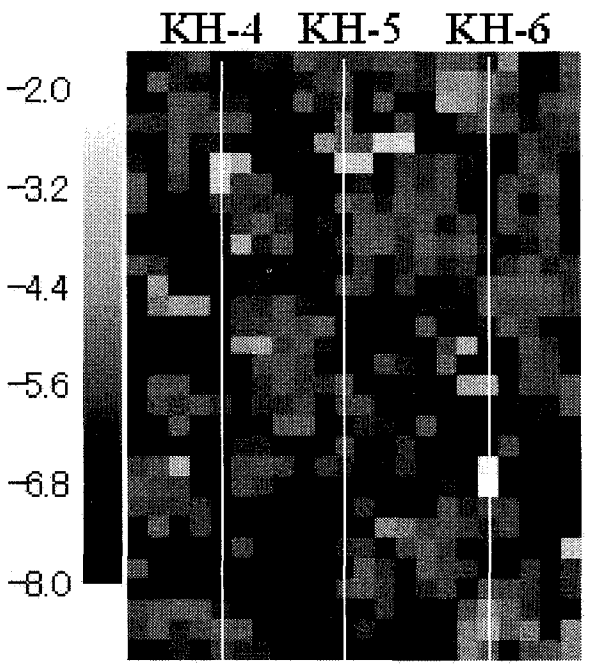

(b) Case 2

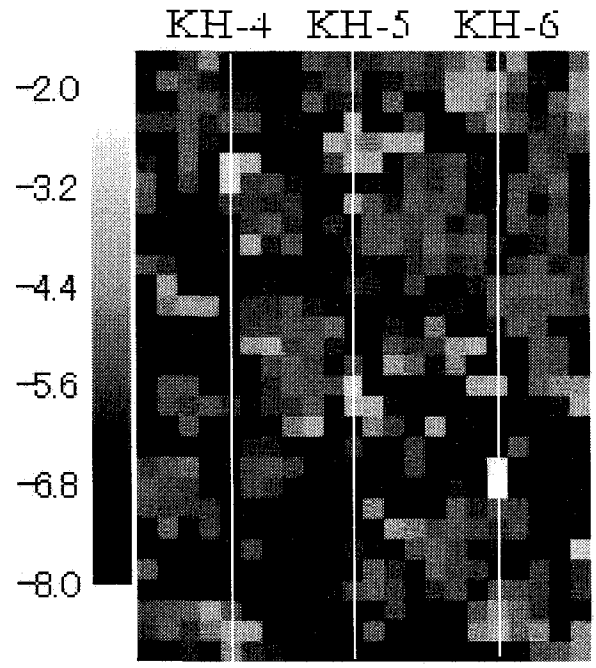

(c) Case 3

(d) Case 4

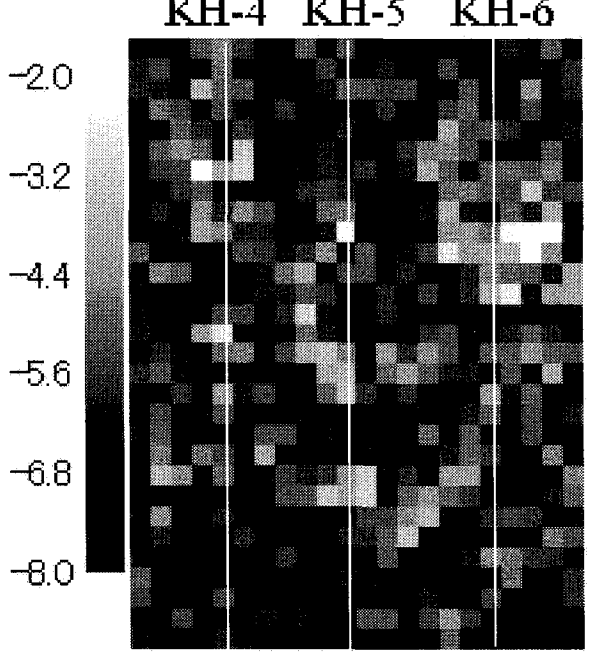

Figure 6: Examples of permeability distribution

(The figure in the legend indicates the logarithm of permeability $(\mathrm{cm} / \mathrm{sec})$ ) 


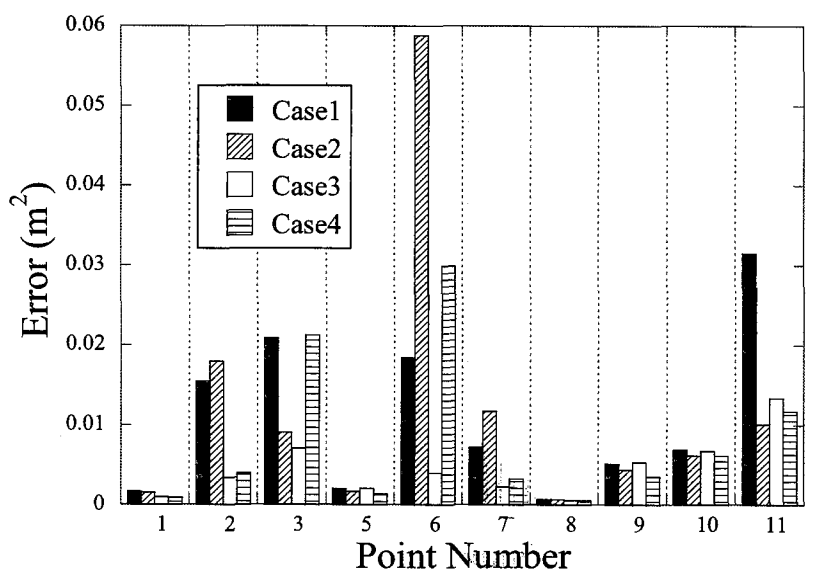

Figure 7: Error at each point

Table 3: Number of hard and soft data and total error of simulation results of each case

\begin{tabular}{cccc}
\hline Case & $\begin{array}{c}\text { Number of } \\
\text { Hard data }\end{array}$ & $\begin{array}{c}\text { Number of } \\
\text { Soft data }\end{array}$ & Errors $\left(\mathrm{m}^{2}\right)$ \\
\hline Case 1 & 15 & 85 & 0.0110 \\
Case 2 & 15 & 85 & 0.0120 \\
Case 3 & 0 & 100 & 0.0046 \\
Case 4 & 0 & 100 & 0.0083 \\
\hline
\end{tabular}

Figure 8 shows comparison of responses at typical measurement points of three different boreholes by Case 1 . As shown in this figure, the measured response at point 10 is relatively high and shows a quick response. This is probably because the path from the source to point 10 has a high permeability. For Case 1, the total error was $0.011 \mathrm{~m}^{2}$.

Considering the entire tendency of the numerical simulations, the ultimate pressures at $\mathrm{KH}-4$ and 7 are relatively well simulated as shown in Figure 8 (a) and (b), while the responses at $\mathrm{KH}-6$ are underestimated as shown in Figure 8 (c). Although the area from the source to point 10 is realized with higher permeability distribution than the area between the source and point 1 in Case1, as shown in Figure 6 (a), it is necessary to realize more permeable paths between source and point 10 .

In Case 2, the assumed relation between permeability and fracture frequency is used. Since a little higher permeability is induced from the relation than the scattergram as shown in Figure 1, the resultant distribution shown in Figure 6(b), shows the lighter color than (a). Figure 9 shows the response at point 10 of KH- 6 by Case 2 . While the response appeared quickly than Case 1, the ultimate pressure is smaller than that in Case 1. The total error is $0.012 \mathrm{~m}^{2}$. In this case, as shown in Figure 7, the large error is caused at the points 2, 6 and 7, which are the points near the source point. As a consequence, the accuracy is not improved by the emphasized relation between permeability and fracture frequency.

In Case 3, the measured permeability is neglected in generating the permeability distribution, while all the measured fracture frequency is conditioned. As well as Case 2,

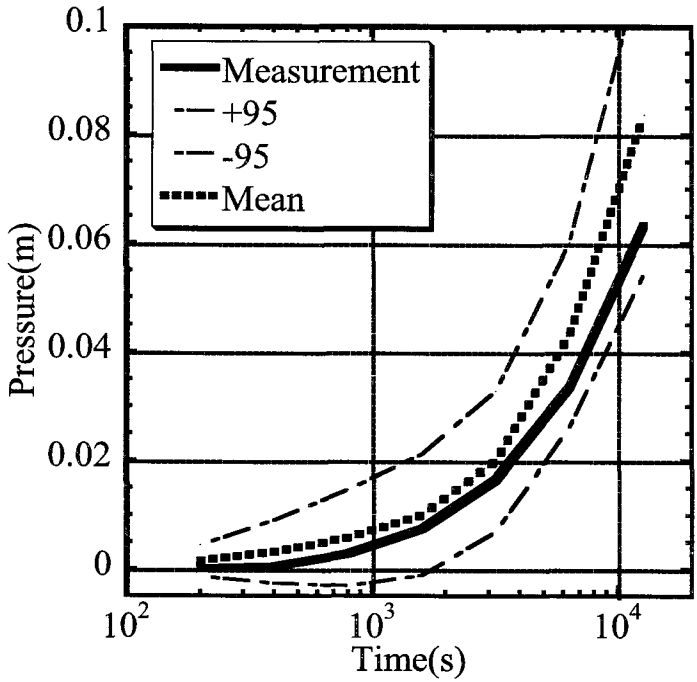

(a) Point 1 of KH-4 by Case 1

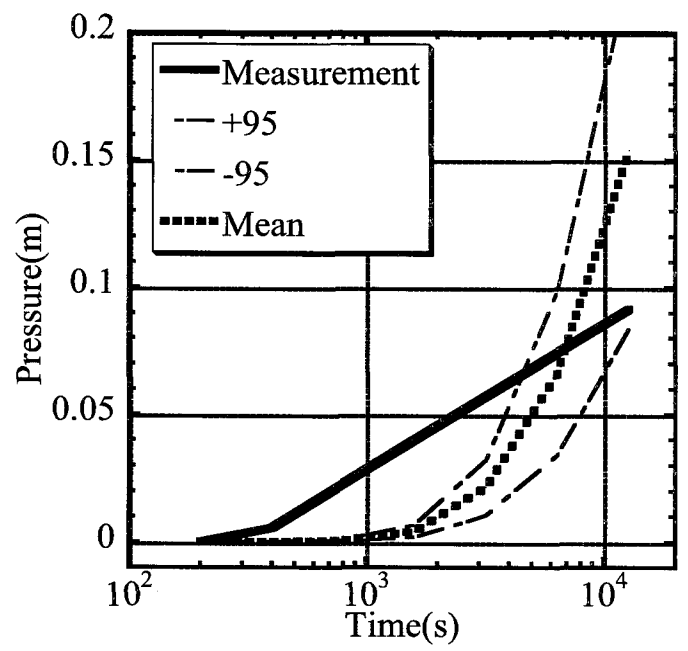

(b) Point 7 of $\mathrm{KH}-7$ by Case 1

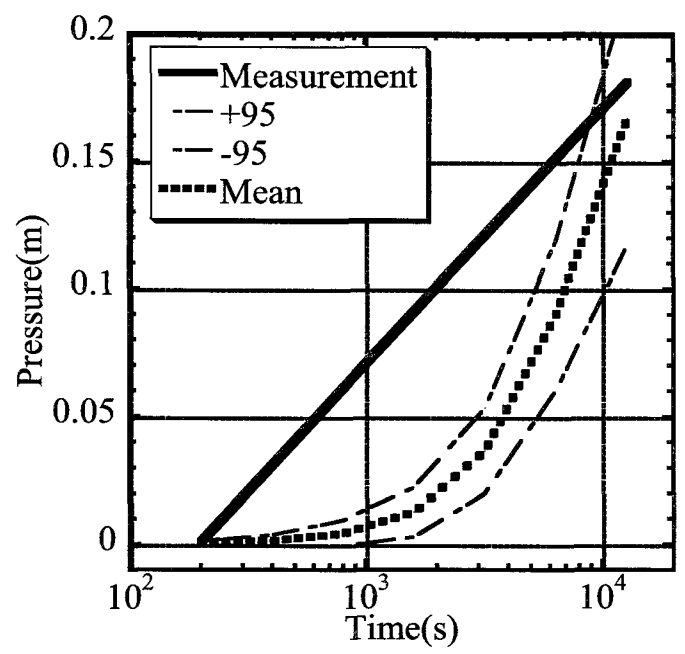

(c) Point 10 of KH- 6 by Case 1

Figure 8: Examples of comparisons of responses by Case 1 (+95 and -95 indicates the range of $95 \%$ confidence of mean value) 


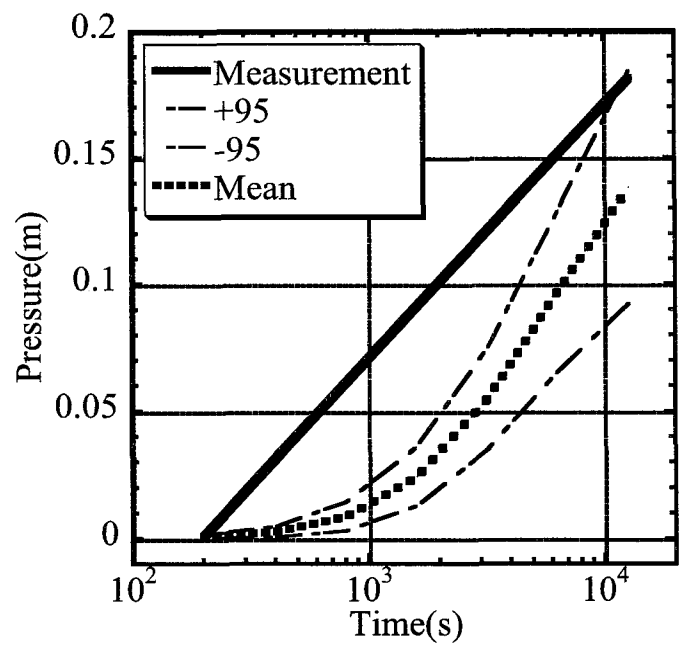

Figure 9: Response at point 10 of $\mathrm{KH}-6$ by Case 2 (+95 and -95 indicates the range of $95 \%$ confidence of mean value)

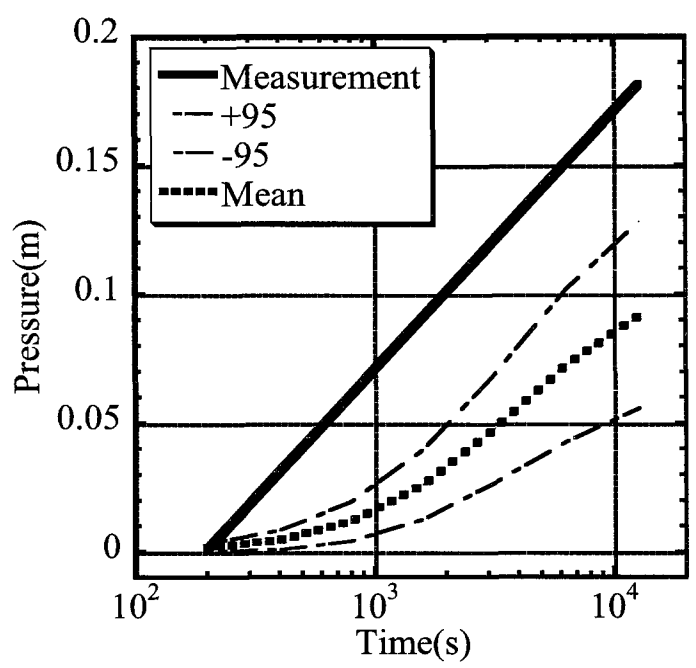

Figure 10: Response at point 10 of KH- 6 by Case 3 ( +95 and -95 indicates the range of $95 \%$ confidence of mean value)

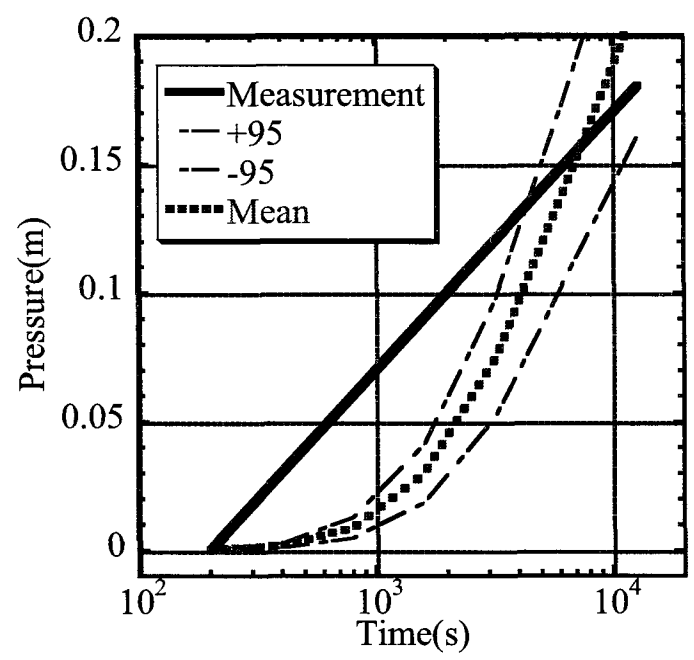

Figure 11: Response at point 10 of KH- 6 by Case 4 ( +95 and -95 indicates the range of $95 \%$ confidence of mean value) the assumed relation between permeability and fracture frequency gives a little high permeability distribution. The number of the part having light color at $\mathrm{KH}-5$ becomes larger than Case 2, as shown in Figure 6 (b) and (c). Figure 10 shows the response at point 10 of $\mathrm{KH}-6$ by Case 3 . While the quick response and small ultimate pressure are observed similarly to Case 2 , the average response is entirely smaller than the measured one. The total error in this case is, however, $0.0046 \mathrm{~m}^{2}$. The accuracy for the entire region is much improved. In particular, the responses in $\mathrm{KH}-7$ are improved as shown in Figure 7.

For Case 4, the permeability distribution is quite random as shown in Figure 6(d), while all the measured fracture frequencies are conditioned. This is because the spatial correlation of fracture geometry data is not considered in the model. Figure 11 shows the result of point 10 by this case. The accuracy for point 10 is the best among the examined cases. This is because the high permeability was distributed around the source and point 10 as shown in Figure 6 (d) in some realizations. The total error is $0.0083 \mathrm{~m}^{2}$. At the points except for points 3 and 6, the error is small as shown in Figure 7. The entire accuracy is improved from Case 1.

\section{Conclusions}

For the case where the permeability measurement is not carried out so much, the ability to use the fracture frequency as soft data to infer the permeability distribution is examined in this study. As a conclusion, the followings are given;

1) The fracture frequency did not correlate well with permeability. Even if the relation was assumed in order to have a good correlation, the result was not improved.

2) The case having the permeability distribution without conditioning the permeability was better than that with conditioning the permeability in this examination case. This is probably because the obtained spatial correlation of permeability was biased to the direction of borehole and thus that was not suitable for the direction normal to the borehole axis. Moreover, the measured permeability was considered to be smaller than the one estimated from the fracture frequency. This is the reason why the result of Case 3 was better than that of Cases 1 and 2.

3) The discontinuous fracture network approach gives the relatively random distribution of fracture frequency. By using the permeability estimated from the random distribution of fracture frequency, the result was better than that of Case 1 while that was not better than the Indicator Simulation without conditioning the permeability (Case 3). It is found from the result to be important to consider the spatial correlation of fracture frequency even if the correlation is biased to a given direction.

It is found from the above results that the Indicator Simulation method without conditioning the permeability is effective to infer the flow path when the number of fracture frequency data is large and that of permeability is small. As a next step, the method to estimate the spatial correlation is 
the subject to examine. As mentioned earlier, the conventional method uses the covariance of hard indicator for that of soft indicator for simplicity. This treatment may be effective when the number of data is not so different for both hard and soft data. In the case when the number of both data is quite different such as the case examined in this study, it is found that the conventional treatment has a problem. In order to improve the accuracy to estimate the permeability distribution with the fracture frequency as soft data, the database about the relation between permeability and fracture frequency has to be made for various rocks.

Moreover, the examination about the anisotropy is another remained subject. While the isotropic distribution was assumed in this study because of the insufficient measurements, modeling of the anisotropic distribution is an important subject.

\section{References}

[1] Bonnet, E., Bour, O., Odling, N., Main, I., Davy, P., Cowie, P., and Berkowitz, B. (2001): Scaling of Fracture Systems in Geological Media, Reviews of Geophysics, 39(3), pp. 347-383.

[2] Dagan, G. (1989): Flow and transport in porous formation, Springer-Verlag, pp.271-289.

[3] Dershowitz, W., Wallmann, P., and Kindred, S.(1991): Stripa Project Technical Report 91-16,SKB.
[4] Deutsch, D.V., and Journel, A.G. (1992): Geostatistical Software Library and User's Guide, Oxford university Press.

[5] Gelhar, L.W. (1993): Stochastic subsurface hydrology, Prentice Hall, pp.199-240.

[6] Ishida, S., Tsuchihara, T., and Imaizumi, M. (2006): Fluctuation of NO3-N in groundwater of the reservoir of the Sunagawa Subsurface Dam, Miyakojima Island ,Japan, Paddy and Water Environment, 4, pp. 101-110.

[7] Journel, A.G. (1989): Fundamentals of geostatistics in Five Les-sons, Short Course in Geology: Vol.8.

[8] Kobayashi, A., Inoue, K., and Aoyama, S. (2005): Improved estimation method of permeability distribution with multiple soft information, Transaction of JIDRE, 39(3), pp. 347-383. (in Japanese).

[9] Kobayashi, A., Fujita, T., and Chijimatsu, M. (1998): Characterization of the hydraulic property and the numerical flow models of fractured rock mass, Soils and Foundations, 38(4), pp. 57-70.

[10] Okuno, T., (1994): Study on the estimation of 3 dimensional distribution of permeability and calculation of ground water flow, Nagoya University Dr. dissertation. (in Japanese).

[11] Yokoi, K., Noguchi, Y., Choh, H., and Hamabe, S. (1991): Geophysical methods applied in underground investigation programs at the Kamaishi Mine, BUTURI-TANSA, 44(6), pp.350-361. (in Japanese).

Discussion open until December 31, 2009 